\title{
Une place pour les savoirs d'expérience en formation des professionnels de la santé
}

Les connaissances biomédicales sont en pleine expansion, dans le sillage d'avancées scientifiques et technologiques, elles-mêmes contemporaines d'investissements massifs dans les services de santé, mis à disposition d'une population vieillissante et atteinte de multiples maladies. Sur le plan épidémiologique, ces avancées ont permis notamment de diminuer la prévalence des maladies infectieuses, en même temps que se développaient considérablement les maladies chroniques.

Parmi les retombées notables d'une telle évolution, on observe qu'il devient plus facile d'élaborer des réponses et des solutions adaptées aux besoins des patients difficiles à guérir et/ou soigner, affectés par des problèmes de santé chroniques particulièrement complexes. Au cours des dernières décennies, l'espérance de vie de la population mondiale n'a cessé d'augmenter, même dans les sociétés où la transition épidémiologique n'est pas encore achevée et où, de ce fait, coexistent et se cumulent les conséquences d'une prévalence et d'une incidence élevées tant des maladies infectieuses que des affections chroniques.

Dans une perspective scientifique «positiviste», ces avancées techno-scientifiques, participent à la construction d'un savoir universel - les lois de l'univers - dont on s'approcherait doucement, avec respect, puisque c'est à partir du développement de ce savoir que nous escomptons la possibilité de comprendre davantage ce qui est en jeu dans «le silence des organes », en allusion à la formule du chirurgien René Leriche ( «La santé, c'est la vie dans le silence des organes »), souvent commentée par Canguilhem [1]. Au cœur d'une telle conception, le «savoir» se trouve distancié, comme s'il devait être entièrement indépendant de nous, loin de nos perceptions qui sont accusées d'introduire un biais entre la réalité telle qu'elle existerait - la vérité - et telle que nous la percevons. Nous subordonnons à la construction d'une telle vérité une façon de raisonner qui, s'agissant du raisonnement clinique, par exemple, cherche à structurer les données des maladies afin d'organiser la pensée clinique, dans la quête d'un haut degré de certitude du raisonnement clinique et de son résultat, dont témoignerait une concordance accrue entre les spécialistes [2].

Cependant, la confrontation du clinicien à des problèmes complexes et mal structurés, caractérisés par le fait que toutes les données ne sont pas disponibles d'emblée ou que la solution n'est pas univoque selon les différents spécialistes concernés, conduit à interroger la notion de certitude et questionne la légitimité de la quête d'une vérité «objective» à laquelle donnerait accès la mobilisation de connaissances quasi exclusivement biomédicales [2].

Les praticiens de la santé témoignent de plus en plus souvent d'un tel inconfort et, en réponse, contestent la perspective selon laquelle leur métier ne consisterait qu'à mettre en pratique ces savoirs qui ne leur appartiennent pas, au motif qu'eux-mêmes ne seraient que des médiateurs de tels savoirs pour le bénéfice de leurs patients et de leurs étudiants. Pour autant, ils ont du mal à s'affranchir de la caution des savoirs scientifiques ou des données probantes, censées confirmer ou invalider les interventions qu'ils mènent auprès de leurs patients. Avec l'avènement et l'hégémonie du courant de l'evidence-based medicine, la quête de preuves ou tout au moins d'arguments solides, validés par les pairs, s'est en effet imposée comme l'élément central du paradigme de la formation et comme la voie à privilégier pour rassurer les professionnels face à la complexité des phénomènes et diminuer les variations de leurs pratiques professionnelles.

Toutefois, la pratique clinique est constamment confrontée à des situations qui mettent à mal le recours à la seule rationalité des savoirs scientifiques au cours du raisonnement clinique, face à des questions qui demeurent sans réponse. Ainsi en est-il des situations de «mauvaise nouvelle» à annoncer concernant l'enfant d'un jeune couple, de décision à prendre quant à l'instauration d'une prise en charge palliative, de confrontation avec le système de croyances d'une famille d'origine culturelle différente de celle des professionnels, de l'insertion dans le système de santé de patients partenaires, ou encore de l'émergence et de la consolidation d'approches en éducation thérapeutique de patients, entre autres, qui confrontent les praticiens à la dimension du sensible au cours de leur exercice. C'est le moment où le recours à leurs propres expériences pour élaborer leurs décisions se fait intuitivement, de façon infra-consciente, sous la force de la répétition de ce qui a été vécu, puisque ni leur formation ni la recherche qui produit les connaissances qu'ils utilisent ne les ont incités au recours légitime aux savoirs d'expérience - ces connaissances hautement personnelles et contextualisées. 
Face au constat d'une formation des professionnels de santé, et singulièrement des médecins, encore majoritairement marqué par l'enseignement de réponses à un répertoire prédéterminé de problèmes présentés de façon unidimensionnelle et structurée, une question émerge: comment peut-on enrichir la formation dans le cadre d'une pédagogie qui conjuguerait les dimensions épidémiologiques, cliniques et humaines inscrites dans tout phénomène liée à la santé?

Dans ces mêmes colonnes, un éditorial de Jacques Roland publié en 2001 [3], alors qu'il était le président en exercice de la Conférence des doyens et des facultés de médecine françaises, insistait sur le besoin de rendre les pédagogies vivantes et sur l'importance de se baser sur ce qui s'inscrit et se construit au fur et à mesure des rencontres avec autrui, comme bases de tout projet de formation professionnelle. Le soignant construit sa compétence sur la base de son vécu. Il n'est pas seulement un transformateur de connaissances en action ou en intentions, il est quelqu'un qui fait des expériences et essaye de les intégrer dans son arsenal de savoirs, construit à la fois par les connaissances formelles et informelles. Le soin et la prise en charge de patients constituent un espace social où les praticiens sont quotidiennement confrontés à une variété d'expériences uniques, qui portent en elles le potentiel d'enrichir l'apprentissage. Il incombe à chacun d'accomplir le travail d'intégration, d'adaptation et de reformulation de ce qui a été vécu, sous la bienveillance et la guidance de ceux qui détiennent un peu plus d'expérience [4].

La formation n'est pas un phénomène qui s'explique, mais qui «se comprend à partir d'une disposition d'entendre ses résonances à travers notre existence » [5]. Aux fins de former des professionnels de la santé capables de naviguer et d'intervenir dans un monde complexe et incertain, il est désormais nécessaire d'inclure la dimension expérientielle dans leur formation. Les apprentissages issus de l'expérience mènent à la mise en relief du rapport qui se construit entre les dimensions formelles et informelles de l'apprentissage [6], donnant fluidité et constance entre les connaissances personnelles développées à partir de l'exercice professionnel, et les connaissances formalisées que l'on partage avec collègues et étudiants.

Les approches que constituent la médecine narrative $[7,8]$ ou les histoires de vie [9] peuvent ainsi être évoquées en tant que démarches qui contribuent à la fois à valoriser la place de la dimension biographique, la construction de savoirs issus de l'expérience, et à ouvrir la formation à la dimension de l'écoute, du dialogue et du sensible, au fur et à mesure que l'étudiant et le professionnel progressent dans leur parcours de développement.

Si la pédagogie médicale est un levier de transformation de systèmes de santé en cours de changement, en lien avec les progrès soulignés à l'ouverture de ce texte, l'évolution des méthodes pédagogiques doit s'articuler autour de multiples registres, d'ordres cognitif, opérationnel, psychologique, social et affectif [10].
Il s'agit d'ouvrir la formation à la dimension de l'incertitude qui fait partie de la pratique médicale moderne, afin d'y ajouter des éléments qui se trouvent en périphérie de la transmission des savoirs formels, de susciter des façons plus éclectiques d'apprendre, et de favoriser la circulation de savoirs pour construire des connaissances davantage en consonance avec la complexité des phénomènes en santé.

En prolongeant la réflexion proposée par Boelen en 2005 [11] sur l'avenir de la pédagogie médicale, nous mettons en avant que 14 ans plus tard, il reste fondamental de répondre aux besoins de la population sur la base de compétences et de valeurs qui permettent d'apprécier les défis actuels, d'améliorer le fonctionnement du système de santé et de collaborer avec les autres acteurs. Dès lors, ne serait-il pas temps d'octroyer une place plus effective aux savoirs d'expérience des étudiants et professionnels de la santé?

Camila ALOISIO ALVES

Attachée temporaire d'enseignement et de recherche, Université Paris-Est Créteil, Post doctorante à l'Université Paris 13 Sorbonne Paris Cité-Laboratoire Experice, axe A, Professeur adjoint à la Faculté de médecine de Petrópolis (Brésil)

Nicolas FERNANDEZ Rédacteur en chef adjoint, Centre de pédagogie appliquée aux sciences de la Santé, Faculté de Médecine, Université de Montréal, Montréal, (Québec, Canada)*

*Mailto : nicolas.fernandez@umontreal.ca

\section{Références}

1. Canguilhem G. Le normal et le pathologique. Paris : Presses Universitaires de France, «Quadrige» (12e édition), 2015.

2. Charlin B. Évaluer la dimension d'incertitude du raisonnement clinique. Pédagogie Médicale 2006;7:5-6.

3. Roland J. La pédagogie dans les Facultés de médecine françaises. Pédagogie Médicale 2001;2:6-8.

4. Delory-Momberger C. De la recherche biographique en éducation. Fondements méthodes pratiques. Paris : Téraèdre (Collection «Autobiographie et éducation »), 2014.

5. Honoré B. Produire sa vie et son histoire-Résonances philosophiques. Lyon: Chronique sociale, 2013.

6. Dominicé P. La formation biographique. Paris : L'Harmat$\tan , 2007$.

7. Charon R. Médecine narrative-Rendre hommage aux histoires de maladies. Paris : Sipayat, 2015.

8. Goupy F, Le Jeunne C. La médecine narrative-Une révolution pédagogique? Paris : Éditions Med-line, 2017.

9. Pineau G, Legrand J-L. Les histoires de vie. Paris: PUF (Collection «Que sais-je?»), 1993.

10. Boelen C. Il y a peut-être un avenir pour la pédagogie médicale... Pédagogie Médicale 2005;6:8-14.

11. Fernandez N. La pédagogie médicale : levier de transformation de systèmes de santé en changement. Pédagogie Médicale 2017;18:45-6. 